\title{
Optimized group velocity control scheme and DNS of decaying compressible turbulence of relative high turbulent Mach number
}

\author{
Xinliang $\mathrm{Li}^{*}, \dagger$, Dexun Fu and Yanwen Ma \\ LNM, Institute of Mechanics, Chinese Academy of Sciences, 100080 Beijing, China
}

\begin{abstract}
SUMMARY
To overcome the difficulty in the DNS of compressible turbulence at high turbulent Mach number, a new difference scheme called GVC8 is developed. We have succeeded in the direct numerical simulation of decaying compressible turbulence up to turbulent Mach number 0.95. The statistical quantities thus obtained at lower turbulent Mach number agree well with those from previous authors with the same initial conditions, but they are limited to simulate at lower turbulent Mach numbers due to the socalled start-up problem. The energy spectrum and coherent structure of compressible turbulent flow are analysed. The scaling law of compressible turbulence is studied. The computed results indicate that the extended self-similarity holds in decaying compressible turbulence despite the occurrence of shocklets, and compressibility has little effects on relative scaling exponents when turbulent Mach number is not very high. Copyright (c) 2005 John Wiley \& Sons, Ltd.
\end{abstract}

KEY WORDS: group velocity control; direct numerical simulation; compressible turbulence; shocklets; extended self-similarity; scaling law

\section{INTRODUCTION}

Shocklets appear in compressible turbulent flow when turbulent Mach number is high enough, and those shock waves in compressible turbulent flow pose severe challenges to DNS [1]. According to Reference [1], most difference schemes designed for steady aerodynamic flow are too dissipative, and even high order ENO schemes are deemed too dissipative. But with non-dissipative schemes, the non-physical oscillations near shock waves will cause in many cases appearance of negative temperatures or pressures in the flow when turbulent Mach

\footnotetext{
${ }^{*}$ Correspondence to: Xinliang Li, LNM, Institute of Mechanics, Chinese Academy of Sciences, No. 15 Beisihuanxi Road, 100080 Beijing, China.

†E-mail: lixl@lnm.imech.ac.cn
}

Contract/grant sponsor: National Natural Science Foundation of China; contract/grant number: 90205025, I9872069 Contract/grant sponsor: NKBRSF; contract/grant number: CG1999032805 
number is high enough. So it is not easy to find a suitable scheme for DNS with high turbulent Mach number compressible turbulent flow and those DNS cases have to be limited to low turbulent Mach number. Porter and Woodward have performed numerical simulation of decaying compressible turbulent flow with initial turbulent Mach number 1.0 [2], but in their simulation Euler equations were solved, and the numerical dissipation was used to mimic the viscous terms.

Samtaney et al. [3] performed DNS of decaying compressible turbulence using the tenthorder Pade scheme. Limited by a 'start-up' problem, the initial turbulent number $\left(M_{\mathrm{t}}\right.$, which is defined by RMS velocity fluctuation and averaged sound speed) is limited to less than 0.5 . $\mathrm{Li}$ et al. [4] performed the DNS of the same flow using seventh-order upwind-biased difference and eighth-order centre difference schemes. The maximum initial turbulent number is improved to 0.7 . Niu [5] performed DNS of this turbulent flow by using optimized three-point fifthorder generalized compact scheme [6], and the initial turbulent Mach number in DNS reaches 0.8. But when the turbulent Mach number is further increased, the 'start-up' problem occurs again. The reason for the 'start-up' problem is the existence of shocklets at high turbulent Mach number. When those shockwaves are strong enough, non-physical numerical oscillations around the shockwave will lead to the failure of the direct numerical simulation.

$\mathrm{Fu}$ and $\mathrm{Ma}[7,8]$ developed a group velocity control theory for shock-capture. According to the theory, a scheme must be 'fast type' at the left side of discontinuity and must be 'slow type' at the right side of the discontinuity (assume the discontinuity propagates towards right), where 'fast type' means that the scheme has positive dispersion error, and 'slow type' means that the scheme has negative dispersion error.

In this paper, a new scheme is advanced by using the group velocity control (GVC) theory. Coefficients of the scheme are optimized for strong shock-wave capture and multi-scale calculation. In the second part of this paper, DNS of decaying compressible turbulent flow with the maximum turbulent number 0.95 is performed. The energy spectrum and coherence of compressible turbulence are analysed, and the scaling law of compressible turbulence is also studied.

\section{THE GVC8 SCHEMES}

Consider the following model equation:

$$
\frac{\partial u}{\partial t}+a \frac{\partial u}{\partial x}=0 \quad \text { assume } a>0
$$

Construct the following upwind-biased scheme:

$$
\begin{aligned}
u_{j}^{\prime}= & \left(a_{1} u_{j+4}+a_{2} u_{j+3}+a_{3} u_{j+2}+a_{4} u_{j+1}+a_{5} u_{j}+a_{6} u_{j-1}+a_{7} u_{j-2}+a_{8} u_{j-3}\right. \\
& \left.+a_{9} u_{j-4}+a_{10} u_{j-5}\right) / \Delta
\end{aligned}
$$

where $u_{j}^{\prime}$ is finite difference approximation of $\partial u / \partial x$ at $x=x_{j}$, and $\Delta=x_{j}-x_{j-1}$ is the mesh spacing. The following control equations of $a_{j}(j=1,2, \ldots, 10)$ can be obtained from the Taylor expansion:

$$
\sum_{j=1}^{10}(5-j)^{k} a_{j}=S_{k} \quad\left(k=0,1, \ldots, K_{\max }\right)
$$




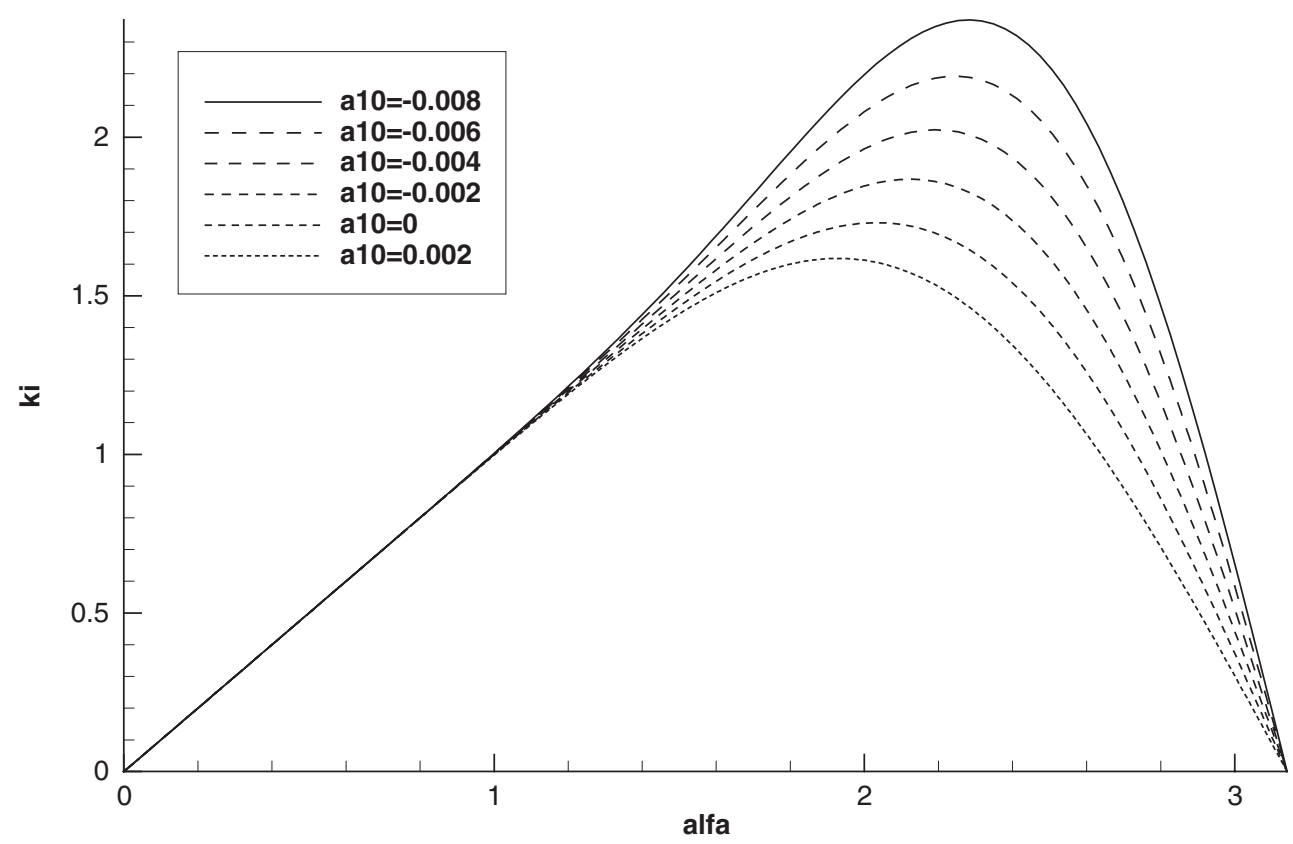

Figure 1. $k_{i}$ varies as a function of $\alpha$.

where $S_{1}=1, S_{k}=0(k \neq 1)$. The approximation of the derivative in (2) has $K_{\max }$ th order accuracy.

Let $K_{\max }=8$, and let $a_{10}$ be a free parameter. When $a_{10}$ is given, all the coefficients can be obtained by solving Equation (3).

With the initial condition $u(x, 0)=\mathrm{e}^{\mathrm{i} k x}$, the exact solution of $(1)$ is $u(x, t)=\mathrm{e}^{\mathrm{i} k(x-a t)}$. With the same initial condition $u\left(x_{j}, 0\right)=\mathrm{e}^{\mathrm{i} k x_{j}}$, solution of the semi-discrete difference approximation equation $\partial u_{j} / \partial t+a u_{j}^{\prime}=0$ is $u\left(x_{j}, t\right)=\mathrm{e}^{-c k_{r} t / \Delta} \mathrm{e}^{\mathrm{i} k\left(x_{j}-c k_{i} t /(k \Delta)\right)}$.

From (2) we have

$$
\begin{aligned}
k_{i}= & -a_{1} \sin 4 \alpha-a_{2} \sin 3 \alpha-a_{3} \sin 2 \alpha-a_{4} \sin \alpha+a_{6} \sin \alpha+a_{7} \sin 2 \alpha+a_{8} \sin 3 \alpha \\
& +a_{9} \sin 4 \alpha+a_{10} \sin 5 \alpha
\end{aligned}
$$

where $\alpha=k \Delta$.

According to GVC theory $[7,8]$, we should have $\mathrm{d} k_{i} / \mathrm{d} \alpha>1$ on the left side of discontinuity, and $\mathrm{d} k_{i} / \mathrm{d} \alpha<1$ on the right side of discontinuity. Figure 1 shows the variation of $k_{i}$ as a function of $\alpha$ for $a_{10}=-0.008,-0.006,-0.004,-0.002,0$ and 0.002 , which shows that when $a_{10}=-0.008,-0.006$ or $-0.004, \mathrm{~d} k_{i} / \mathrm{d} \alpha>1$, and when $a_{10}=-0.002,0$ or $0.002, \mathrm{~d} k_{i} / \mathrm{d} \alpha<1$ in the low wave number region. We can choose different $a_{10}$ on the left side and right side of discontinuity.

Following is the GVC8 scheme in detail:

Let

$$
u_{j}^{\prime}=\left(F_{j+1 / 2}-F_{j-1 / 2}\right) / \Delta
$$


where

$$
\begin{aligned}
F_{j+1 / 2}= & b_{1} u_{j+4}+b_{2} u_{j+3}+b_{3} u_{j+2}+b_{4} u_{j+1}+b_{5} u_{j}+b_{6} u_{j-1}+b_{7} u_{j-2} \\
& +b_{8} u_{j-3}+b_{9} u_{j-4}
\end{aligned}
$$

and

$$
b_{1}=a_{1}, \quad b_{k}=b_{k-1}+a_{k} \quad(k=2,3, \ldots, 9)
$$

$a_{k}$ are the coefficients in Equation (2).

If we substitute $j-1$ for $j$ in (6), we can get $F_{j-1 / 2}$.

In general, we may choose the free parameter as follows:

$$
\begin{aligned}
& a_{10}=a_{10}^{(1)} \text { when }\left|\Delta u_{j-1 / 2}\right|<\left|\Delta u_{j+1 / 2}\right| \text { (at the left side of discontinuity) } \\
& a_{10}=a_{10}^{(2)} \text { when }\left|\Delta u_{j-1 / 2}\right| \geqslant\left|\Delta u_{j+1 / 2}\right| \text { (at the right side of discontinuity) }
\end{aligned}
$$

where $\Delta u_{j}=u_{j+1 / 2}-u_{j-1 / 2}$.

When $a_{10}^{(1)}$ and $a_{10}^{(2)}$ are given, the coefficients $b_{k}$ can be obtained by solving Equations (2) and (7). Expression (5) together with (6) is the eighth-order GVC scheme (GVC8) for the case $a>0$ in (1). In the same way, we can construct the GVC 8 schemes for the case $a<0$.

The GVC8 schemes have eighth-order accuracy at the smooth area, and have seventh-order at the extreme points of $u$.

\section{PARAMETER SELECTION AND OPTIMIZATION}

There are two free parameters $\left(a_{10}^{(1)}\right)$ and $\left(a_{10}^{(2)}\right)$ in the GVC8 scheme. We will select those two parameters to improve the shockwave-capture capability and the resolution of the small scales.

Consider the one-dimensional Sod shock tube problem [9]. The control equations are onedimensional Euler equations

$$
\begin{aligned}
& \frac{\partial U}{\partial t}+\frac{\partial F}{\partial x}=0, \quad x \in[0,1], t \geqslant 0 \\
& U=[\rho, \rho u, e]^{\mathrm{T}} \\
& F=\left[\rho u, \rho u^{2}+p, u(e+p)\right]^{\mathrm{T}} \\
& e=\frac{p}{\gamma-1}+\frac{\rho u^{2}}{2}
\end{aligned}
$$

with the following initial conditions:

$$
\begin{aligned}
& u=0, \quad \rho=\rho_{\mathrm{s}}, \quad p=p_{\mathrm{s}}, \quad x<\frac{1}{2} \\
& u=0, \quad \rho=0.125, \quad p=0.1, \quad x \geqslant \frac{1}{2} \quad \text { at } t=0
\end{aligned}
$$


Table I. Maximum $p_{\mathrm{s}}$ for different GVC8 schemes.

\begin{tabular}{lrcc}
\hline$\left(a_{10}^{(1)}, a_{10}^{(2)}\right)$ & $p_{\text {s } \max }$ & $\left(a_{10}^{(1)}, a_{10}^{(2)}\right)$ & $p_{\text {s max }}$ \\
\hline$(-0.00128,0.0042)$ & 8.5 & $(-0.006,-0.004)$ & 3.5 \\
$(-0.006,0.001)$ & 17.0 & $(-0.008,-0.003)$ & 5.0 \\
$(-0.006,0.002)$ & 12.0 & $(-0.006,-0.006)$ & 2.5 \\
$(-0.006,0.0015)$ & 18.5 & $(-0.0015873,-0.0015873)$ & 1.5 \\
\hline
\end{tabular}

Let $p_{\mathrm{s}}=\rho_{\mathrm{s}}$, which means that the temperature is the constant $T_{\mathrm{s}}=\gamma$, and higher $p_{\mathrm{s}}$ denotes the stronger shockwaves. We solve this Sod problem numerically by using GVC8 scheme with grid number 128, and three-stage total variation diminishing (TVD)-type Runge-Kutta method [4] for time advance. When $p_{\mathrm{s}}$ is high enough, which means that the shock is strong enough, the computation will overflow (usually negative pressure or temperature occurs during the computation). Define $p_{\mathrm{s} \max }$ as the maximum of $p_{\mathrm{s}}$, so that the numerical simulation can be performed.

Table I shows $p_{\mathrm{s} \max }$ for different GVC8 schemes. From Table I, we find that when the parameters are chosen as $\left(a_{10}^{(1)}, a_{10}^{(2)}\right)=(-0.006,0.001)$ or $(-0.006,0.0015)$, the corresponding $p_{\mathrm{s} \max }$ is the highest. These values $\left(a_{10}^{(1)}, a_{10}^{(2)}\right)$ can be considered as a good choice.

The resolution of small scales is an important problem in the simulation of turbulence. Schemes will be too dissipative if the resolution of small scales is not good enough. If $a_{10}^{(1)}=a_{10}^{(2)}$, the GVC8 scheme has eighth-order accuracy; otherwise, the scheme has only seventh-order accuracy. So we can infer that the numerical error will increase as the difference between $a_{10}^{(1)}$ and $a_{10}^{(2)}$ increases. This can be seen from the following example:

Let $u=\sin 16 x, x \in[0,2 \pi]$, and $u_{j}^{\prime}$ be the differential approximation of $\partial u / \partial x$ by using GVC8 scheme with mesh number 128. Define the error err $=\max \left|u_{j}^{\prime}-16 \cos 16 x_{j}\right|$. Figure 2 shows the error as a function of $a_{10}^{(1)}$ and $a_{10}^{(2)}$. From the figure we can see that, in the range of this figure, the error increases as the difference between $a_{10}^{(1)}$ and $a_{10}^{(2)}$ increases.

Considering both capability of shock-capture and the resolution of small scales, we choose $\left(a_{10}^{(1)}, a_{10}^{(2)}\right)=(-0.006,0.001)$.

As a summary, the GVC8 scheme can be described as follows:

The derivative $\partial u / \partial x$ in (1) is approximated as

$$
u_{j}^{\prime}=\left(F_{j+1 / 2}-F_{j-1 / 2}\right) / \Delta
$$

where

$$
\begin{aligned}
F_{j+1 / 2} & =\left\{\begin{array}{ll}
\sum_{k=1}^{9} b_{k}^{+} u_{j+5-k} & \text { when } a>0 \\
\sum_{k=1}^{9} b_{k}^{-} u_{j+6-k} & \text { when } a<0
\end{array} \quad(a \text { is a coefficient in (1) })\right. \\
b_{k}^{+} & = \begin{cases}b_{k}^{(1)^{+}} & \text {when }\left|\Delta u_{j-1 / 2}\right| \leqslant\left|\Delta u_{j+1 / 2}\right| \\
b_{k}^{(2)^{+}} & \text {when }\left|\Delta u_{j-1 / 2}\right|>\left|\Delta u_{j+1 / 2}\right|\end{cases}
\end{aligned}
$$




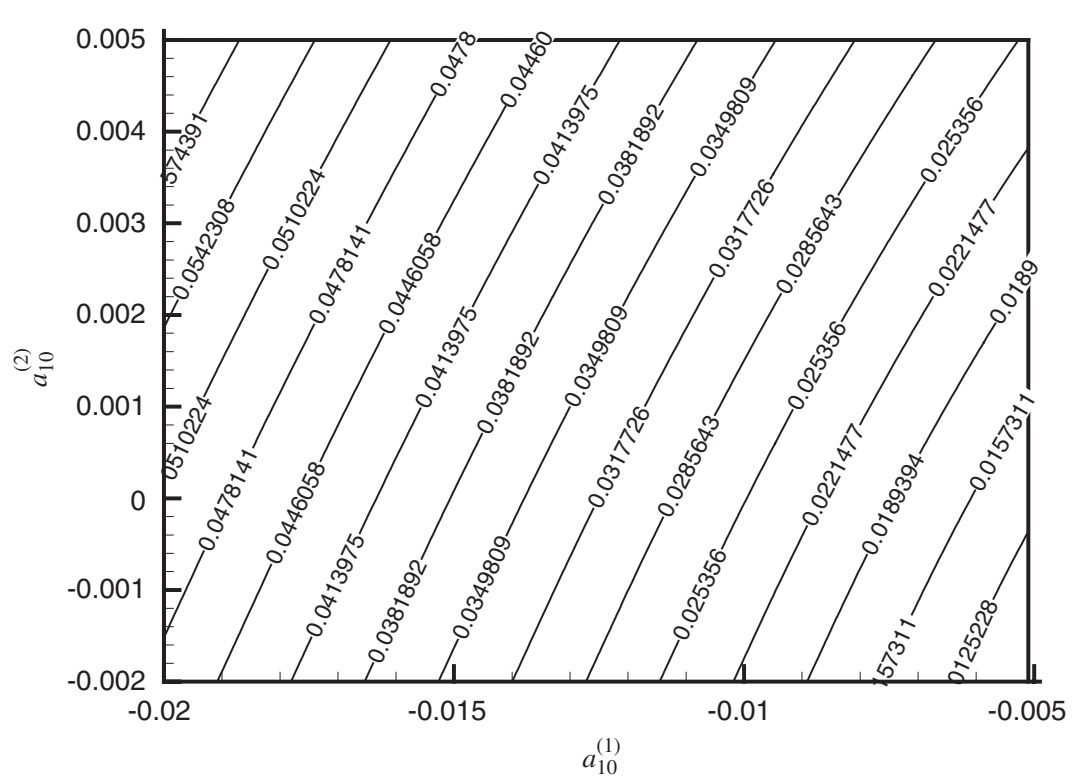

Figure 2. Numerical error varies as a function of $a_{10}^{(1)}$ and $a_{10}^{(2)}$.

Table II. $b_{k}^{(1)^{+}}$and $b_{k}^{(2)^{+}}$.

\begin{tabular}{cccccccccc}
\hline$k$ & 1 & 2 & 3 & 4 & 5 & 6 & 7 & 8 & 9 \\
\hline$b_{k}^{(1)}$ & $\frac{17}{7000}$ & $-\frac{283}{21000}$ & $\frac{53}{21000}$ & $\frac{6269}{21000}$ & $\frac{4429}{4200}$ & $-\frac{10531}{21000}$ & $\frac{4253}{21000}$ & $-\frac{361}{7000}$ & $\frac{3}{500}$ \\
$b_{k}^{(2)}$ & $-\frac{4}{875}$ & $\frac{893}{21000}$ & $-\frac{4063}{21000}$ & $\frac{14501}{21000}$ & $\frac{2371}{4200}$ & $-\frac{2299}{21000}$ & $\frac{137}{21000}$ & $\frac{31}{7000}$ & $-\frac{1}{1000}$ \\
\hline
\end{tabular}

$$
\begin{aligned}
b_{k}^{-} & =\left\{\begin{array}{l}
b_{10-k}^{(2)}{ }^{+} \\
b_{10-k}^{(1)}+
\end{array} \quad \text { when }\left|\Delta u_{j-1 / 2}\right|<\left|\Delta u_{j+1 / 2}\right|,\right. \\
\Delta u_{j} & =u_{j+1}-u_{j-1}
\end{aligned}
$$

$b_{k}^{(1)^{+}}$and $b_{k}^{(2)^{+}}$are given in Table II.

\section{NUMERICAL TEST}

\subsection{The test of efficiency}

To test the efficiency of this scheme, we calculate the difference approximation of $\partial u / \partial x$ in the same computer by using GVC8 scheme, ninth-order ordinary upwind-biasing difference scheme 
Table III. The numerical error and CPU times by using difference schemes.

\begin{tabular}{lcc}
\hline Numerical schemes & Numerical error & CPU time (s) (run 1000 times) \\
\hline GVC8 & $2.73 \times 10^{-9}$ & 0.25 \\
WENO5 & $1.55 \times 10^{-5}$ & 3.36 \\
UD9 & $2.13 \times 10^{-10}$ & 0.16 \\
\hline
\end{tabular}

Table IV. Numerical conditions.

\begin{tabular}{lcc}
\hline Case & Numerical method & Grid number \\
\hline T1 & WENO5 & 4000 \\
T1 & WENO5 & 8000 \\
T2 & GVC8 & 400 \\
T3 & WENO5 & 400 \\
T4 & UD9 & 400 \\
\hline
\end{tabular}

(UD9) or fifth-order weighted essentially non-oscillatory scheme (WENO5) [10], separately. $u=\cos (100 x)(x \in[0,2 \pi])$ is chosen as the test function with mesh grid number $N=10000$. The numerical error and CPU time (run 1000 times) are shown in Table III (the computer has a $2.0 \mathrm{GHz}$ Pentium4 CPU).

This table shows that the numerical accuracy of GVC8 is much higher than that of WENO5, and CPU time of the calculation using GVC8 is only $\frac{1}{13}$ of that using WENO5. From this table, we can see that the numerical accuracy and efficiency of GVC8 are near those of UD9. As shown in this section, GVC8 scheme can calculate flow with strong shockwave like WENO schemes; therefore, GVC8 scheme is very suitable for the DNS of compressible turbulence with stronger shockwaves.

\subsection{One-dimensional Shu-Osher shock tube problem}

This problem is a one-dimensional shock tube problem [11] with oscillations, the control equation is one-dimensional Euler equation (8), and the initial condition is

$$
\begin{array}{lr}
\rho=3.857, \quad u=2.629, \quad p=10.333 & \text { when } x<0.1 \\
\rho=1+A \sin (\omega x), \quad u=0, \quad p=1 & \text { when } x \geqslant 0.1
\end{array}
$$

where $A=0.3, \omega=40$.

We performed numerical simulation of this problem by using the three schemes in Table III. Flux vector splitting is adopted, and three-stage TVD-type Runge-Kutta method is used for time advance. The time step is $\Delta t=0.00002$ for all cases, which is rather small to release time error. The numerical conditions are shown in Table IV.

Cases $\mathrm{T} 1$ and $\mathrm{T}^{*}$ are performed at very dense grid. Figure 3 shows plots of density at $t=0.2$ for cases $\mathrm{T} 1$ and $\mathrm{T} 1^{*}$, and the difference between the two results is very small, so the result of case T1 can be considered as an 'exact solution' of this problem. 


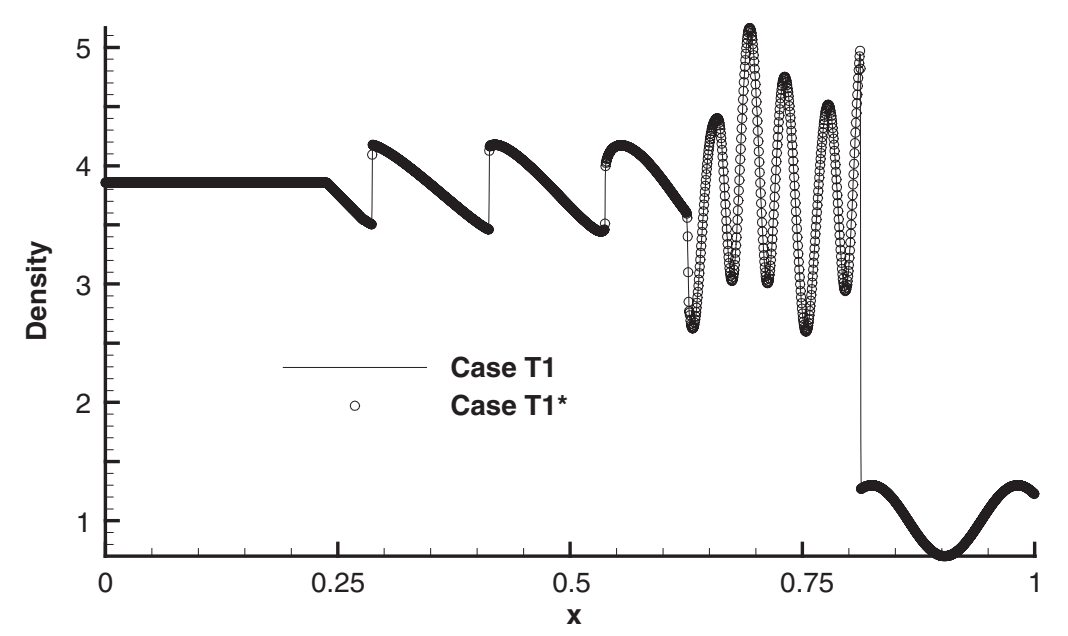

Figure 3. Plot of density for cases $\mathrm{T} 1$ and $\mathrm{T} 1^{*}$.

Case T4 became float overflow at the first several time steps, which means that UD9 scheme cannot be used in this problem.

Plots of density at $t=0.2$ for cases T1, T2 and T3 are shown in Figures 4(a)-(c), where Figures 4(b) and (c) are the partially enlarged drawings. From those figures, we can see that the result of T2 is more close to the exact solution, which means that GVC8 scheme can capture shocks and fluctuations more correctly.

\section{DIRECT NUMERICAL SIMULATIONS OF COMPRESSIBLE DECAYING ISOTROPIC TURBULENCE AT RELATIVE HIGH TURBULENT MACH NUMBERS}

In this section, we perform DNS of compressible decaying turbulent flow [3,4]. Flux vector splitting is adopted for the convection terms that are discretized by GVC8, WENO5 or UD7 (seventh-order upwind-biasing difference scheme [4]). Eighth-order central difference scheme is used to discretize viscous terms, and three-stage TVD-type Runge-Kutta method is used for time advance.

The velocity starts with a specified spectrum that is initially divergence free and the energy spectrum is $E(k)=A k^{4} \exp \left(-2 k / k_{0}^{2}\right)$, where $k$ is the wave number, $k_{0}$ is the wave number at which the spectrum peaks, and $A$ is the amplitude, which is a constant number. All initial thermodynamic quantities (pressure, density and temperature) are constant. Periodic boundary conditions and uniform meshes are used in $x, y$ and $z$ directions.

The Reynolds number based on Taylor scale is defined as $R e_{\lambda}=u^{\prime} \lambda\langle\rho\rangle /\langle\mu\rangle$, where $u^{\prime}=$ $\left\langle\left(u_{1}^{2}+u_{2}^{2}+u_{3}^{2}\right) / 3\right\rangle^{1 / 2}$ is root mean square (RMS) velocity fluctuation and $\lambda^{2}=u^{\prime 2} /\left\langle\left(\partial u_{1} / \partial x_{1}\right)^{2}\right\rangle$ is Taylor scale. The symbol $\langle\bullet\rangle$ denotes a volume average over the computational domain at a fixed time instant.

The turbulent Mach number is defined as $M_{\mathrm{t}}=\left\langle u_{1}^{2}+u_{2}^{2}+u_{3}^{2}\right\rangle^{1 / 2} /\langle c\rangle$, where $c$ is the local sound speed. 


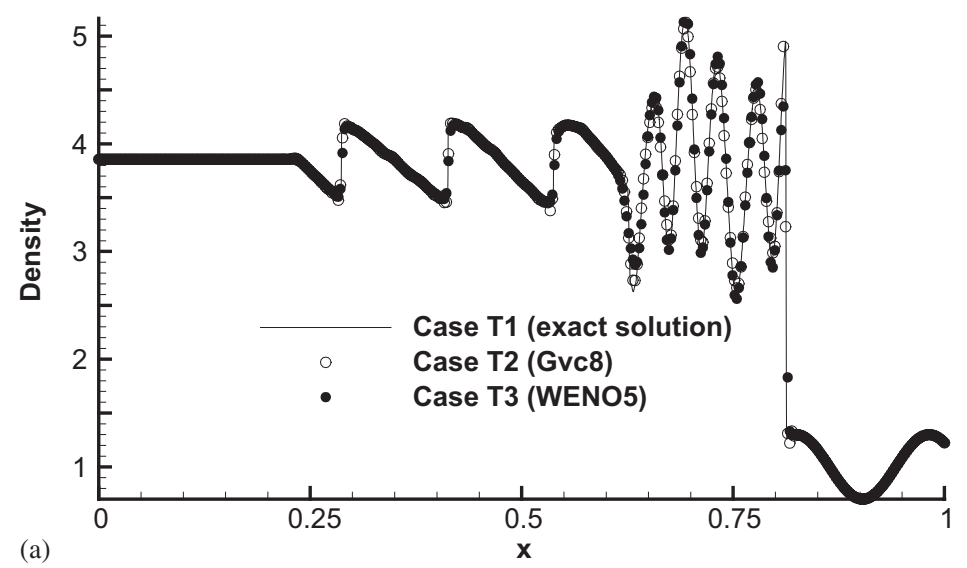

(a)
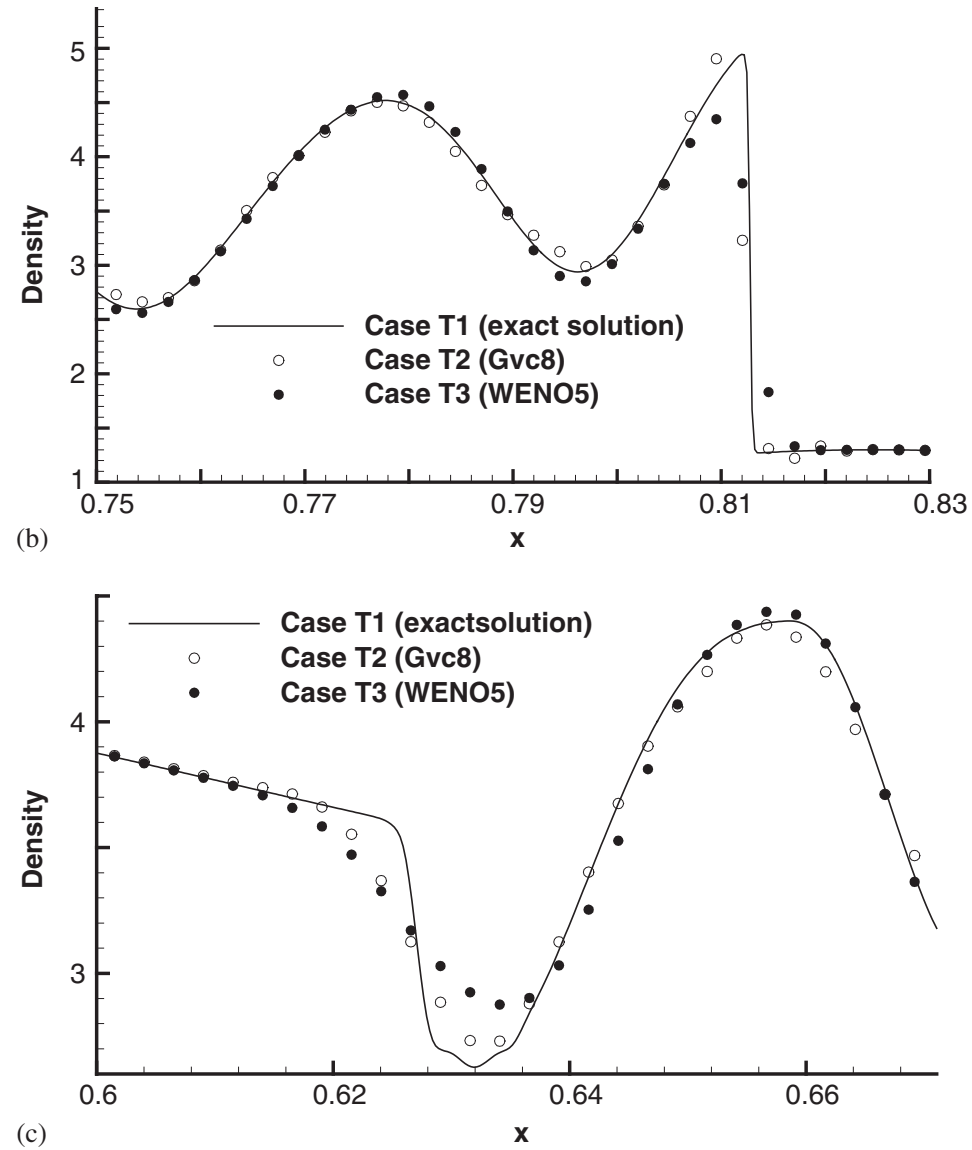

Figure 4. (a) Plot of density for cases T1, T2 and T3; (b) plot of density of cases T1, T2 and T3 (partial enlarged drawing); and (c) plot density of cases T1, T2 and T3 (partial enlarged drawing). 
Table V. The computational conditions of each DNS case.

\begin{tabular}{lrlcc}
\hline Case & $R e_{\lambda}$ & $M_{\mathrm{t}}$ & Scheme & Mesh size \\
\hline D1 & 72 & 0.5 & GVC8 & $128^{3}$ \\
D2 & 72 & 0.8 & GVC8 & $128^{3}$ \\
D3 & 72 & 0.9 & GVC8 & $128^{3}$ \\
D4 & 72 & 0.95 & GVC8 & $128^{3}$ \\
D5 & 72 & 0.5 & WENO5 & $128^{3}$ \\
D6 & 72 & 0.9 & GVC8 & $256^{3}$ \\
E1 & 153 & 0.6 & GVC8 & $256^{3}$ \\
E2 & 153 & 0.8 & GVC8 & $256^{3}$ \\
E3 & 153 & 0.6 & WENO5 & $256^{3}$ \\
F1 & 153 & 0.3 & UD7 & $256^{3}$ \\
F2 & 153 & 0.6 & UD7 & $256^{3}$ \\
\hline
\end{tabular}

Table VI. The computing performance of each case.

\begin{tabular}{lccc}
\hline Case & Running CPU & CPU Time (s/step) & Computer \\
\hline D1-D4 & 8 & 34 & SMC cluster \\
D5 & 8 & 86.7 & SMC cluster \\
D6 & 32 & 135 & LSSC1 \\
E1-E2 & 64 (in 32-nodes) & 6.8 & LSSC2 \\
E3 & 64 (in 32-nodes) & 37 & LSSC2 \\
F1-F2 & 32 & 105 & LSSC1 \\
\hline
\end{tabular}

${ }^{*}$ Case F2 are also performed on 64 CPUs in 32-nodes of LSSC2, the performance is about $5.7 \mathrm{~s} /$ step.

Table V shows the DNS cases. From this table, we can see that the maximum initial turbulent Mach number is 0.95, which is much higher than that in Reference [3]. The Reynolds numbers (based on Taylor scale and RMS velocity fluctuation) are 72 and 153. Cases F1 and F2 can be found in Reference [4]. For case D1-D6, $A=0.00013, k_{0}=8$, and for other cases, $A=0.00055, k_{0}=6$.

The program was coded by using MPI Fortran77. In the program, long messages are cut into short messages to avoid deadlock and improve the efficiency. Performance of each case is shown in Table VI where SMC cluster is a 16-node PC cluster of the SMC Center in Tsinghua University, and the CPU of SMC cluster is PIII 733. LSSC1 is a 128-node PC cluster of State Key Laboratory of Scientific and Engineering Computing (LSEC), and the CPU is PIII 550. LSSC2 is a 256-node 512-CPU cluster of LSEC, and CPU is Pentium 4 at 2.0 GHz.

Figure 5 shows the time history of normalized average kinetic energy $K(t) / K(0)$ and velocity derivative skewness $S_{3}$ for Case D1, where

$$
K(t)=1 / 2\left\langle\rho u_{i} u_{i}\right\rangle, \quad S_{3}=\left\langle\left(\frac{\partial u_{1}}{\partial x_{1}}\right)^{3}\right\rangle /\left\langle\left(\frac{\partial u_{1}}{\partial x_{1}}\right)^{2}\right\rangle^{3 / 2}
$$




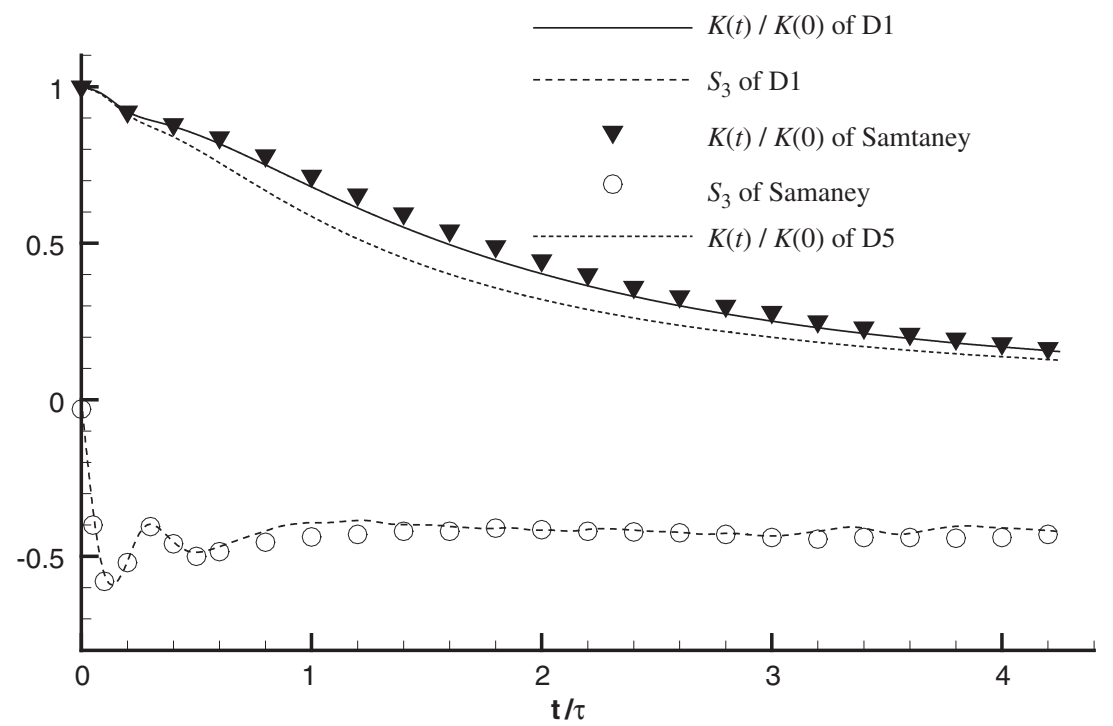

Figure 5. Normalized turbulent kinetic energy and skewness factor of velocity derivative $\left(M_{\mathrm{t}}=0.5\right)$.

The horizontal ordinate of Figure 5 denotes normalized time $t / \tau$, where

$$
\tau=\sqrt{\frac{32}{A}}(2 \pi)^{1 / 4} k_{0}^{-7 / 2}
$$

is large-eddy-turnover time at $t=0$ [3].

The symbols in Figure 5 show the results of Reference [3], which agree well with those of this paper. This means that the dissipative error of the GVC8 scheme can be ignored in the mesh resolution (the result does not seem to be over-dissipative). Velocity derivative skewness, which is a high-order statistical quantity, agrees well with those of Reference [3], and this verified our results.

Figure 6 shows the time history of normalized turbulent Mach number and normalized RMS of density fluctuations $\rho_{\mathrm{rms}} / M_{\mathrm{t}}^{2}(0)$, where $\rho_{\mathrm{rms}}=\left\langle\rho^{2}\right\rangle^{1 / 2}$. The symbols in the figure denote the corresponding results of Reference [3], which agree well with those of this paper. The results of case D5 (by using WENO5) are also shown in Figure 6, which seemed too dissipative.

Figure 7 shows the time history of normalized turbulent kinetic energy and velocity derivative skewness of case D2. Figure 8 shows the time history of normalized turbulent Mach number and RMS of density of case D2. The symbols in these two figures are the results of Reference [5] at the same initial conditions. These two figures show that the results of GVC8 schemes agree well with the result of Reference [5], which verified our results.

Figure 9 shows the time history of normalized turbulent kinetic energy of cases D3 (mesh size: $128^{3}$ ) and D6 (mesh size: $256^{3}$ ), from which we can see that the results of case D3 agree well with those of D6. This comparison shows that our result is reliable. Figure 10 shows the time history of normalized turbulent kinetic energy of D1-D4, from which we can see that the turbulent kinetic energy decays faster as the initial turbulent Mach number 


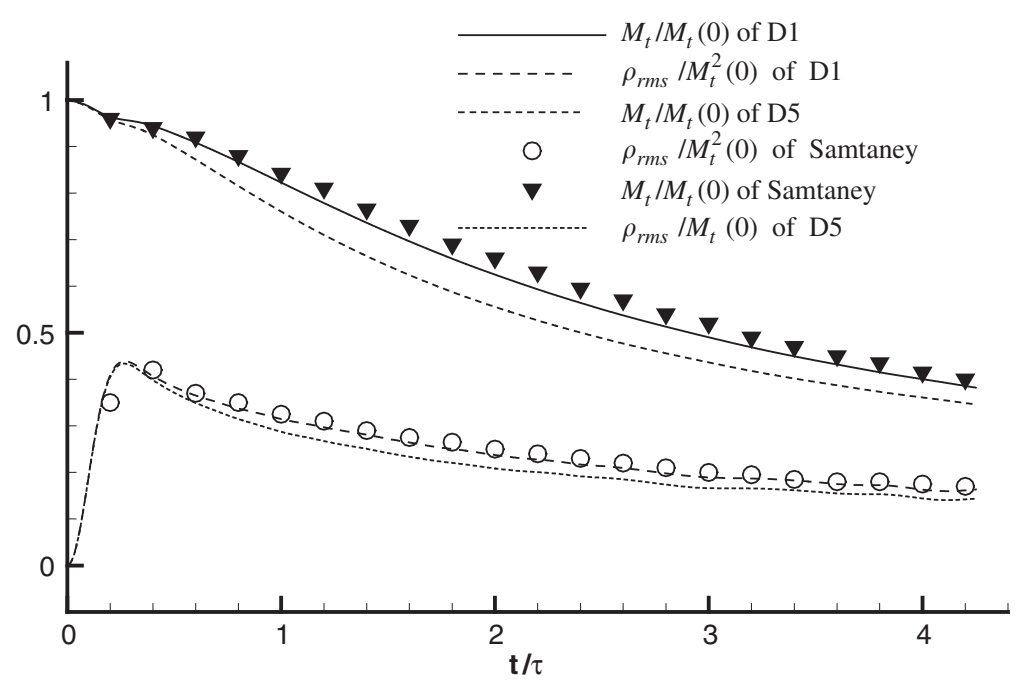

Figure 6. Normalized turbulent Mach number and normalized RMS of density fluctuation $\left(M_{\mathrm{t}}=0.5\right)$.

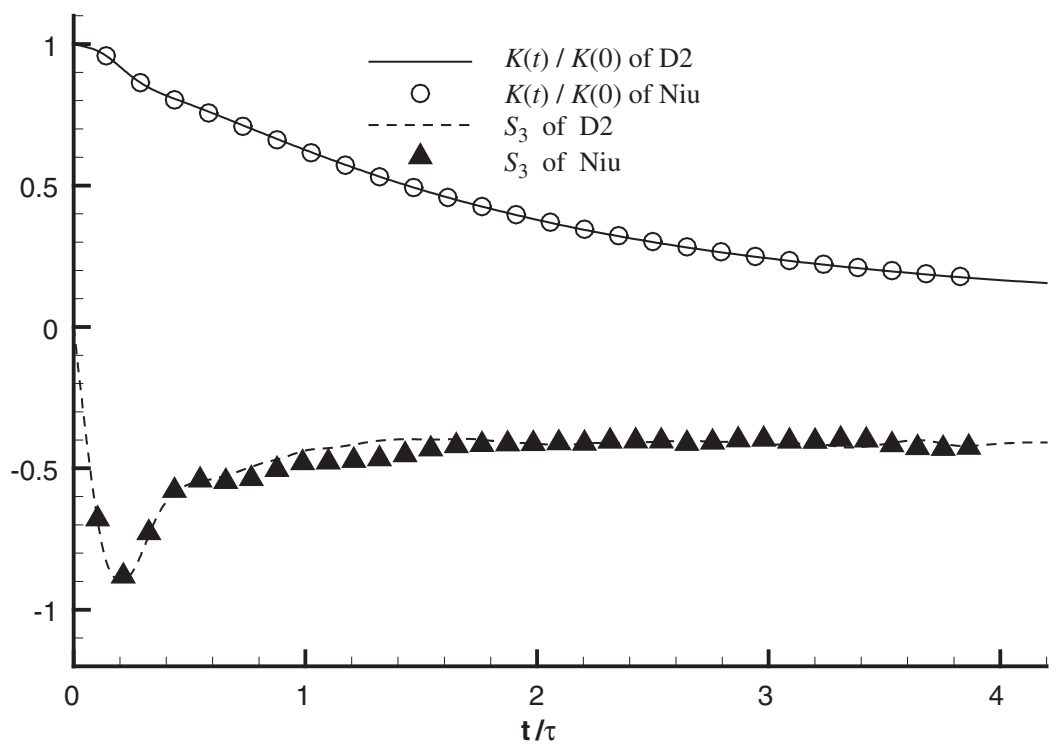

Figure 7. Normalized turbulent kinetic energy and velocity derivative skewness $\left(M_{\mathrm{t}}=0.8\right)$.

increases, and this means that more kinetic energy will be transformed into intrinsic energy as turbulent Mach number increases.

Figure 11 is the time history of normalized average kinetic energy $K(t) / K(0)$ and velocity derivative skewness $S_{u}$ for Cases E1, E3 and F2. The initial Reynolds number, initial turbulent Mach number and mesh size are the same for these three cases $\left(R e_{\lambda}=153, M_{\mathrm{t}}=0.6\right.$ and mesh 


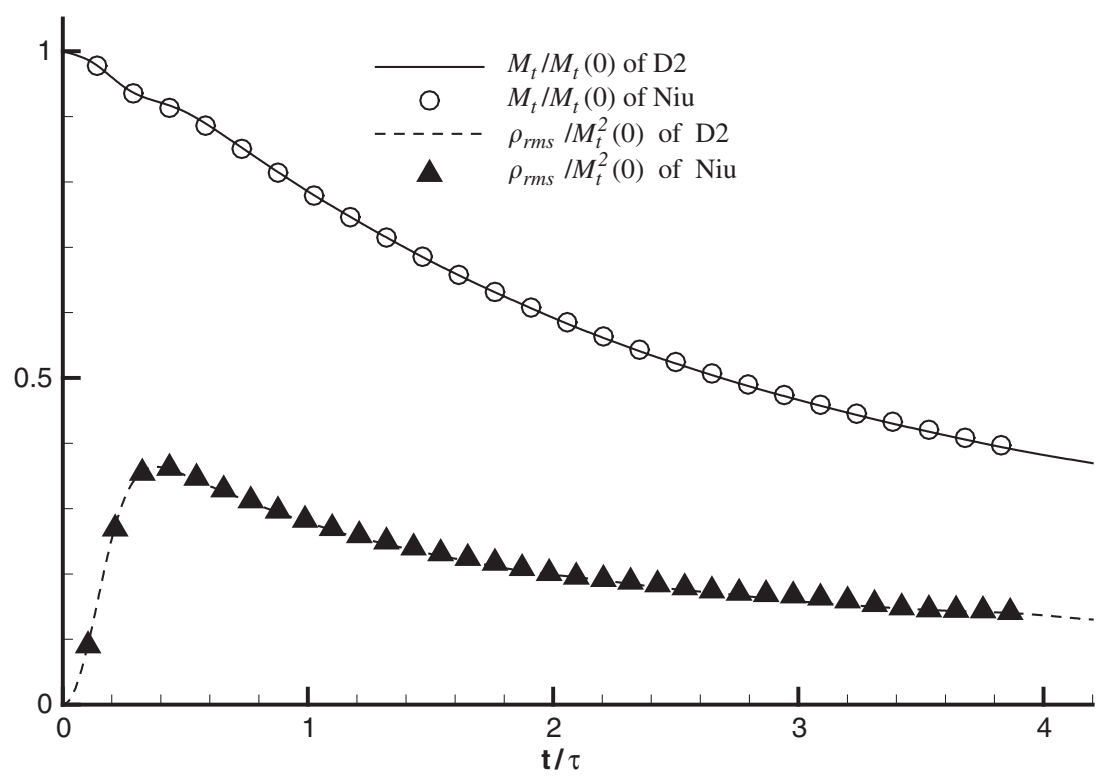

Figure 8. Normalized turbulent Mach number and normalized RMS density fluctuation $\left(M_{\mathrm{t}}=0.8\right)$.

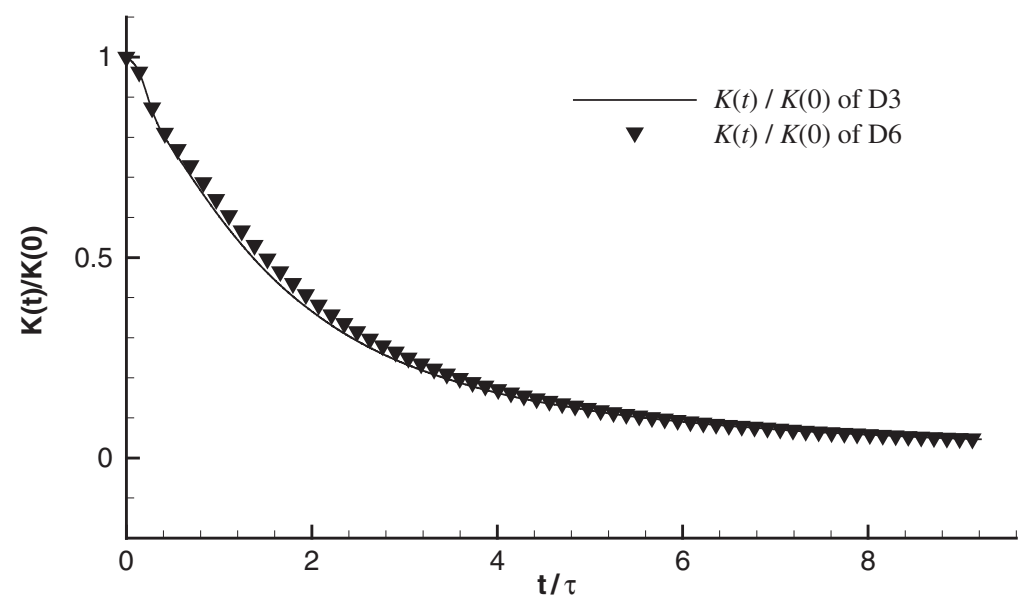

Figure 9. Time history of normalized turbulent kinetic energy of D3 and D6.

size is $256^{3}$ ). The numerical method is different for these cases. GVC8, UD7 and WENO5 schemes are used in these three cases separately. Case F2 has been tested and verified in Reference [4]. According to this figure, the result of case E1 agrees well with that of F2, and the kinetic energy of case E3 seems to be a little over-dissipative. This figure shows that the results of GVC8 are better than those of WENO5.

Figures 5-11 validate our computation. 


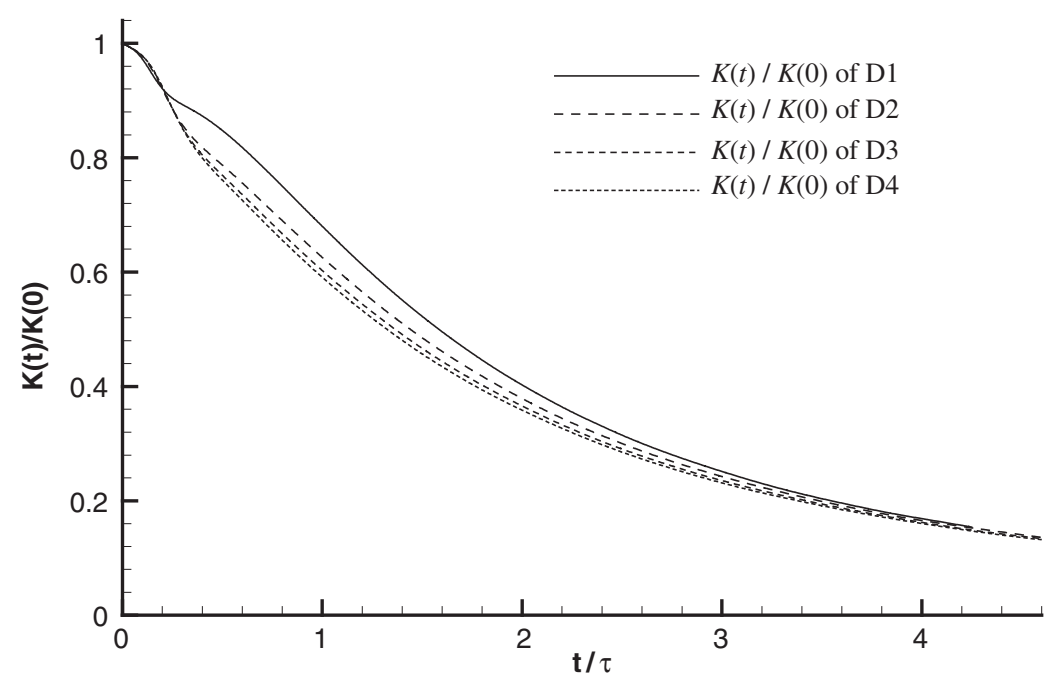

Figure 10. Time history of normalized turbulent kinetic energy of computing case D1-D4.

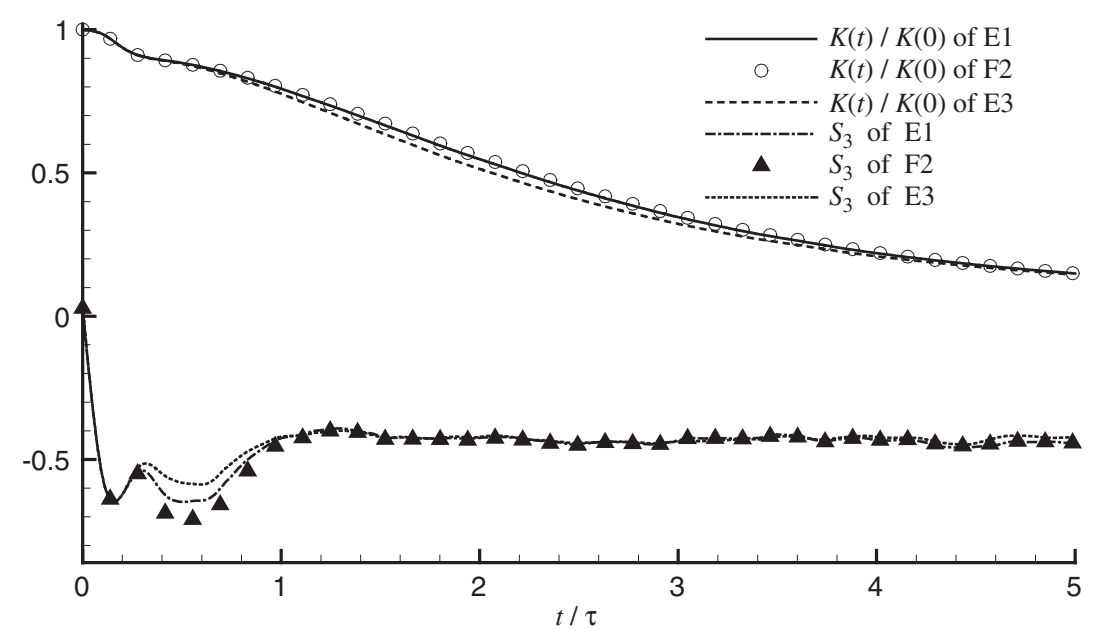

Figure 11. Normalized turbulent kinetic energy and velocity derivative skewness for computing case E1 (using GVC8), E3 (using WEN05) and F2 (using UD7).

According to Reference [3], in the computation, the turbulence fluid after $t / \tau=0.5$ ( $\tau$ is a large eddy turn-over time) may be considered to be realistic. We will study the flow field of those computation cases at $t / \tau \geqslant 1$ which are shown in Table VII. FD6 is flow field at $t / \tau=1$ of computing Case D6. FE1, FE2 and FF1 are flow fields at different times of Case E1, E2 and F1 and the Reynolds number of those three flow fields is nearby the same.

Figure 12 shows the energy spectra of flow field FE2, FE1 and FF1. Because Reynolds number is not high, the inertial range of the energy spectra is short. 
Table VII. Flow field number and flow parameters.

\begin{tabular}{lcccr}
\hline Flow field number & Case & Normalized time & $R e_{\lambda}$ & $M_{\mathrm{t}}$ \\
\hline FD6 & D6 & $t / \tau=1$ & 33.05 & 0.72 \\
FE1 & E1 & $t / \tau=1.192$ & 66.935 & 0.54 \\
FE2 & E2 & $t / \tau=1$ & 66.937 & 0.70 \\
FF1 & F1 & $t / \tau=1.304$ & 66.923 & 0.275 \\
\hline
\end{tabular}

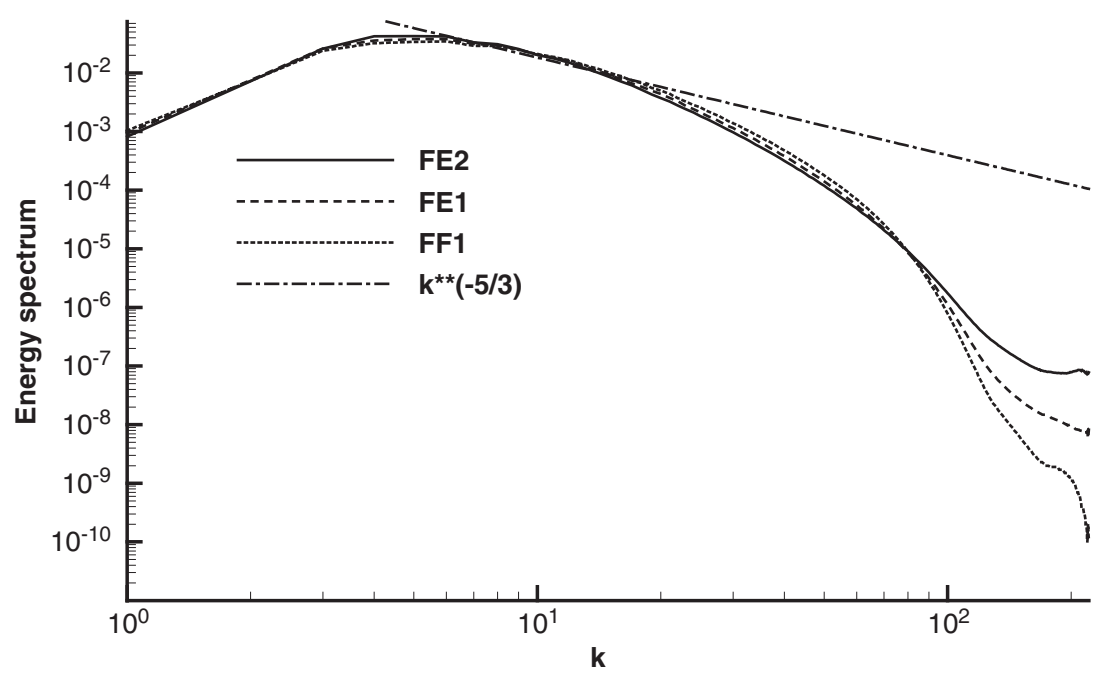

Figure 12. Energy spectrum of flow field FE2, FE1 and FF1.

Figure 13 shows the contour surface of normalized second invariant of the velocity gradient tensor $\tilde{Q}$ of flow field FE2, FE1 and FF1. The visualized region is $\frac{1}{64}$ of the whole computing domain, where $\tilde{Q}=Q / Q_{\mathrm{rms}}$, and $Q=-\left(S_{i j} S_{i j}-W_{i j} W_{i j}\right)$ is the second invariant of velocity gradient tensor, where $S_{i j}=\left(u_{i, j}+u_{j, i}\right) / 2$ and $W_{i j}=\left(u_{i, j}-u_{j, i}\right) / 2$. The contour surface of $Q$ is often used to show the coherent structures [12]. From this figure, we can see there are many tube-like structures in these flows, and the effect of compressibility on these coherence structures is not significant.

Shocklets occur in the flow when turbulent number is high enough [3]. According to the computation data, we have a very high local Mach number in some areas even when the averaged one is not so high. For example, in the computation case D6, when $t / \tau=1$, the averaged Mach number is 0.653 , but the maximum local Mach number of this flow fluid is 3.276. High local Mach number is probably because of the intermittency of turbulence. The authors conjecture that the intermittency of turbulence is an important reason for the shocklets creation.

Extended self-similarity (ESS) is an important development in the measurement of the scaling exponents. It states that the velocity structure function of any order $p$ depends on the structure functions of order $q$ (usually 3) in a much better power law: $\left\langle\left|\delta u_{l}\right|^{p}\right\rangle \sim\left\langle\left|\delta u_{l}\right|^{q}\right\rangle^{\zeta_{p, q}}$, where $\zeta_{p, q}=\zeta_{p} / \zeta_{q}$ is called the relative scaling exponent. ESS holds well even when the 


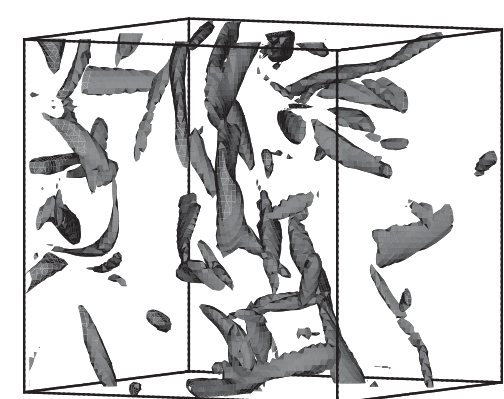

FE2

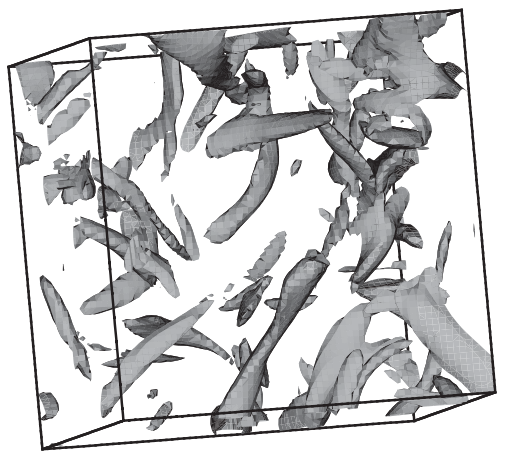

FE1

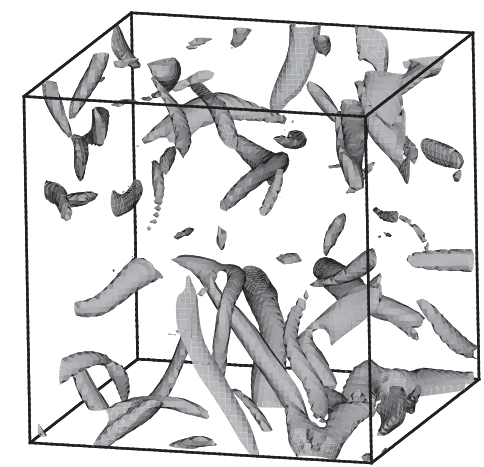

FF1

Figure 13. Contour surface of normalized second invariant of the velocity gradient tensor for flow field FE2, FE1 and FF1 $(\tilde{Q}=1.6$ for all the three flow field $)$.

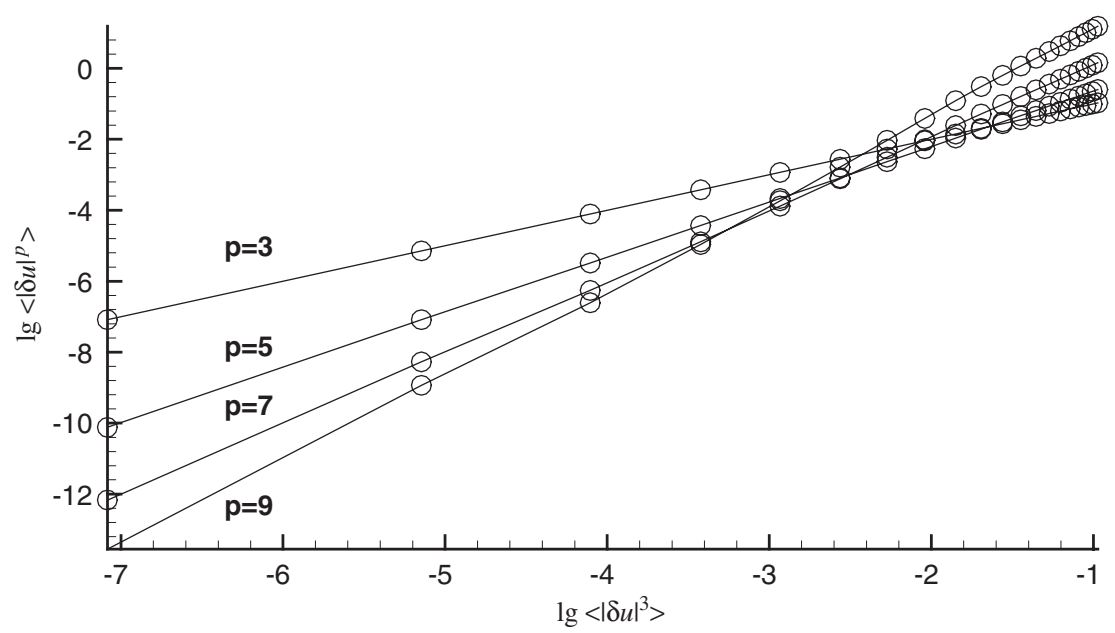

Figure 14. ESS of the flow field FD6. 


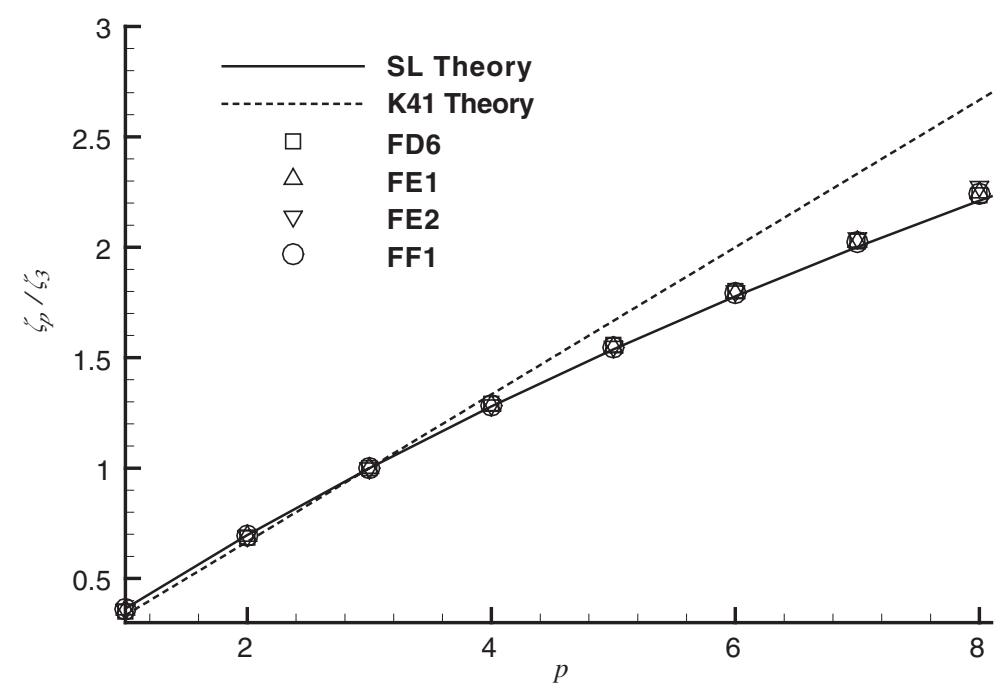

Figure 15. The relative scaling exponents.

Reynolds number is relatively low and there is no clear inertial range in turbulent flow. Figure 14 shows the plot of $\lg \left\langle\left|\delta u_{l}\right|^{p}\right\rangle$ as a function of $\lg \left\langle\left|\delta u_{l}\right|^{3}\right\rangle$ in flow field FD6. For all $p, \lg \left\langle\left|\delta u_{l}\right|^{p}\right\rangle$ is shown as a strict linear function of $\lg \left\langle\left|\delta u_{l}\right|^{3}\right\rangle$. This figure shows clearly the existence of ESS in this compressible turbulence flow in spite of the existence of the shocklets. The slope rates of the lines in this figure are the relative scaling exponents $\zeta_{p} / \zeta_{3}$. Least squares techniques are used to obtain these exponents. Figure 15 shows relative scaling exponents of flow fields FD6, FE1, FE2 and FF1. This figure shows that the difference between the relative scaling exponents of those flow fields is small, which shows the universality of the scaling exponents. When turbulent Mach number is not very high, compressibility has little effect on scaling exponents. The dashed line in this figure shows the predicted values by K41 theory and the solid line shows the predicted value by the SL theory [13], and this figure shows that our numerical result agrees well with the SL theory.

\section{CONCLUSION}

In this paper, a new scheme named GVC8 is developed for shock capture. To test this scheme, DNS of compressible isotropic turbulence is performed. The conclusions of this paper are as follows:

1. GVC8 scheme has high accuracy for multi-scale problem and can capture stronger shockwaves. This scheme is suitable for DNS of compressible turbulent flow of relatively high turbulent Mach number. Additionally, the efficiency of this scheme is high.

2. More energy transfer to small scales as compressibility effects increase.

3. There are many tube-like coherence structures in the compressible isotropic turbulent flow, and it seems that compressibility has little effect on these tube-like structures. 
4. ESS holds in the compressible turbulent flow in spite of the presence of shocklets, and compressibility has little effect on scaling exponents when turbulent Mach number is not very high.

\section{ACKNOWLEDGEMENTS}

The authors would like to thank the State Key Laboratory of Scientific and Engineering Computing (LSEC) for providing computer time. The authors thank Professor Zhuang Fenggan for the helpful discussion on this paper, Professor Zhang Linbo of LSEC for help in programming, and Professors Chen Shiyi and He Guowei for help in settling the initial condition of DNS. This project was supported by National Natural Science Foundation of China (90205025, 19872069) and NKBRSF (CG1999032805).

\section{REFERENCES}

1. Moin P, Mahesh K. Direct numerical simulation: a tool in turbulence research. Annual Fluid Mechanics 1998; 30:539-578.

2. Porter DH, Woodward PR. Inertial range structure in decaying compressible turbulent flow. Physics of Fluids 1998; 10(1):237-245.

3. Samtaney R, Pullin DI, Kosovic B. Direct numerical simulation of decaying compressible turbulence and shocklet statistics. Physics of Fluids 2001; 13(5):1415-1430.

4. Li XL, Fu DX, Ma YW. Direct numerical simulation of compressible isotropic turbulence. Science in China, Serial A 2002; 45(11):1452-1460.

5. Niu XL. Shock-capturing generalized compact scheme and application in DNS of turbulence. Doctoral Dissertation, Tsinghua University, Beijing, China, 2002.

6. Shen MY, Niu XL, Zhang ZB. The three-point fifth-order accurate generalized compact scheme and its applications. Acta Mechanica Sinica 2001; 17(2):142-150.

7. Fu DX, Ma YW, Kobayashi T et al. Nonphysical oscillations in numerical solutions-reason and improvement. Computational Fluid Dynamics Journal 1996; 4(4):427-450.

8. Fu DX, Ma YW. A high order accurate difference scheme for complex flow. Journal of Computational Physics 1997; 134:1-15.

9. Sod G. Journal of Computational Physics 1978; 27(1):1-31.

10. Liu XD, Osher S, Chan T. Weighted essentially non-oscillatory schemes. Journal of Computational Physics 1994; 115:200-212.

11. Shu CW, Osher S. Efficient implementation of essentially non-oscillatory shock-capturing schemes I. Journal of Computational Physics 1988; 77:439-471.

12. Tanahashi M, Miyauchi T, Ikeda J. Scaling law of coherent fine scale structure in homogeneous isotropic turbulence. Proceedings of the 11th Symposium on Turbulent Shear Flows, vol. 1, 1997, 4-17-4-22.

13. She ZS, Leveque E. Universal scaling law in fully developed turbulence. Physical Review Letters 1994; 72(3):336-339. 